\title{
DETECTING FLOW METER DRIFT BY USING ARTIFICIAL NEURAL NETWORKS
}

\author{
M. BEN SALAMAH ${ }^{1}$, E. PALANEESWARAN ${ }^{2}$, M. SAVSAR ${ }^{3}$ \& M. EKTESABI ${ }^{2}$ \\ ${ }^{1}$ The Electrical Power Department, The Higher Institute for Energy, The Public Authority for \\ Applied Education and Training (PAAET), Kuwait. \\ ${ }^{2}$ Faculty of Engineering and Industrial Sciences, Swinburne University of Technology, \\ Hawthorn, Victoria, Australia. \\ ${ }^{3}$ Industrial and Management Systems Engineering Section, the College of Engineering \& Petroleum, \\ Kuwait University, Kuwait.
}

\begin{abstract}
In this paper, artificial neural networks (ANNs) were used to assess the performance of flow meters used in industrial water supply. These flow meters are susceptible to drift, a condition causing them to give erroneous readings that are inconsistent with the actual flow. A simulation of industrial water flow to the industrial consumers was made. This simulation contained both healthy and drifting flow meter readings. ANN was built and trained on the simulated data. At the time of testing, the ANN developed was correct $89.52 \%$ of the time in determining the status of the flow recorded by a flow meter.
\end{abstract}

Keywords: artificial neural network, flow meter drift, industrial water supply, statistical process control.

\section{INTRODUCTION}

The importance of water cannot be overemphasized. Because of this, people realized the importance of controlling, organizing, and regulating its use. No sensible controlling, organizing, regulating, and planning can be done without accurate measurement. Hence, the abundance of flow meters in any water system.

Accurate measurement arrangements are required for effectively managing water infrastructure regimes. Flow meters can be subject to specific failures such as erroneous readings and breakdown. Inaccurate measurements affect the controlling, organizing, and regulating processes of the water system and planning. Measures for avoiding and mitigating such flow meter problems include: calibration, repair, and replacement. Flow meter calibration generally involves checking against accurate standards to determine any appreciable deviations and correcting for errors. Yet, this calibration is not a one time transaction as it does not end the problem forever.

Flow meter calibration is often neglected by the operation and maintenance staff of a water system. Drift is an error in measurement, which can increase with time. Ben Salamah et al. [1] outlined a statistical process control (SPC)-based flow meter drift detection method for the industrial water use regime. Realizing the usefulness of artificial neural network (ANN) modeling for forecasting and classification objectives, an extension of this research considered specific applications of ANN modeling with improved SPC frameworks for flow meters. This paper presents some key findings from the application of ANN models for detecting flow meter drifts.

\section{LITERATURE REVIEW}

Cheng [2] provided an alternative to SPC methods. The SPC methods, namely the Shewhart and CUSUM control schemes, were replaced by ANNs. The neural network architecture that Cheng used was the three-layer fully connected feed-forward network with back propagation. For the inputs for his network, Cheng used both numerical and symbolic inputs. For the numerical inputs, Cheng used a string of 16 past data. This string of data, called window, did not use the original values of the process. 
Rather, it used transformed values; the transformed values were obtained by a coding scheme. For the symbolic input, Cheng used one run rule (an explanation for run rules will follow). The run rule that he used was the one regarding exceeding $\pm 3 \sigma$ (where $\sigma$ is the process's standard deviation). Cheng reported that ANNs were $20-40 \%$ faster in detecting small process changes than the traditional Shewhart and CUSUM control schemes. Later, Cheng [3] used two types of pattern recognizers based on different neural network architectures: a multi-layer perceptron trained by back-propagation and a modular neural network. Cheng [3] noticed that the modular neural network provided better recognition accuracy than back-propagation when high strong interference effects existed.

One important application of ANNs is in pattern recognition (for a general introduction to pattern recognition that includes using ANNs, the reader is referred to Friedman and Kandel [4]; for the more specialized subject of pattern recognition by ANNs only, the reader is referred to Bishop [5]). Consequently, there have been many papers on the subject of using ANNs for pattern recognition of SPC methods. Anagun [6] used a multi-layered neural network trained with a back propagation algorithm for pattern recognition of control charts. A method called histogram representation was employed. Hassan et al. [7] used ANNs for pattern recognition of SPC charts and compared two methods of data input with the ANNs: raw data and statistical features of data. The ANN with the statistical features performed better than the one with raw data.

Pacella et al. [8] applied the adaptive resonance theory (ART) neural networks in their work. They have presented a fuzzy ART neural system for quality control. The purpose of the system was the detection of abnormal process behavior. Pecella et al. mentioned that the advantage of their system over other neural techniques was that it did not require previous knowledge about the abnormal patterns or their mathematical models or probability distribution functions.

In practice, the Shewhart control chart is used with what is called 'Supplementary Run Rules'. These rules indicate when to investigate a process when the points plotted on the Shewhart chart exhibit certain behaviors. The run rules can be thought of as primitive pattern recognition methods. Koutras et al. [9] presented the subject of Shewhart control charts that are supplemented with additional rules. Yasui et al. [10] introduced two additional run rules. According to their work, a process might be considered out of control if

1. Two of three successive observations exceed \pm 2.0698 sigma control limits.

2. Two successive observations exceed \pm 1.9322 sigma control limits.

\section{PRODUCTION PROCESSES AND THEIR QUALITY ASSURANCE}

In this section, the nature of production processes is described. These processes have the tendency to deteriorate with time which will decrease the quality of products. Consequently, there is a need for quality-assurance methods to prevent this. The most common method is the Shewhart chart.

Any process aiming to produce a consistent and constant product, such as a reinforcing bar of a certain diameter or a brick of certain dimensions or a chemical product with specific properties, etc. would usually produce this product according to the standard required but with some deviation. This deviation is usually expected of any process and the allowance made for deviation is called the tolerance, $\tau$, of the process or product. If the quality we are interested in is called $X$ and the desired value of $X$ is $\bar{X}$, the plot of $X$ against time, $t$, would look like what is shown in Fig. 1. In this figure, the vertical axis shows the magnified area of the desired value, $\bar{X}$, and its tolerances.

$X$ at a certain time, is designated by $X_{t}$. For example, we would have $X_{1}, X_{2}, X_{3}$ corresponding to values of $X$ at times $t=1, t=2, t=3$, respectively. The values of $X_{t}$ oscillate closely around the desired value, $\bar{X}$. 


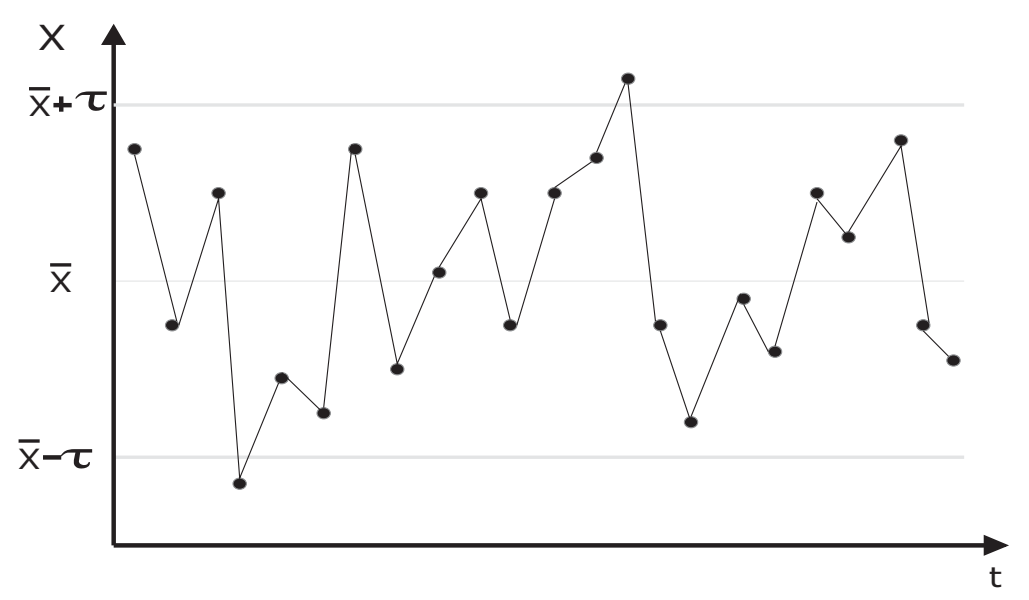

Figure 1: A process varying over time.

If the process is in good condition, the central limit theorem states that 'if the sample size $\mathrm{n}$ is large then the population of all possible sample means approximately has a normal distribution, no matter what probability distribution describes the population sampled' [11]. In an established process, $n$ is usually large. This would make the samples of the process cluster around the target or desired value $\bar{X}$. If the values of the samples, $X$, are plotted against time, they will oscillate closely around the desired value, $\bar{X}$. This would actually make the desired or target value, $\bar{X}$, the average of the process.

When the process is faulty, the assumption of normality is no longer valid. In this case, the measured values of $X_{t}$ no longer cluster around $\bar{X}$ and start drifting away from it. Hence, when constructing a control (Shewhart) chart, which will be shortly explained, Norton [12], suggested the following:

'If interest is in controlling the process to keep measurements as close as possible to a target value, $T$, then the $y$ intercept of the center line should be $T^{\prime}$.

\subsection{Introduction to the Shewhart chart}

The Shewhart chart is one of the most widely used methods for ensuring that a product is produced according to what is desired. It is a quality assurance method achieved by monitoring the behavior of a process. More precisely, it is about observing the extent to which a process stays close to its mean (or strays away from it). To observe this, the chart needs to use the mean of the process or quality, $\bar{X}$, and its standard deviation, $\sigma$. The Shewhart chart is made up of seven lines: one line at the mean of the process and a line at the following values: $\pm \sigma, \pm 2 \sigma, \pm 3 \sigma$. Figure 2 shows the Shewhart chart made against the process of Fig. 1.

Several run rules have been developed to utilize the Shewhart chart. The purpose of these rules is to prevent the process from deviating beyond what is permitted. An example of a run is the following:

Rule 1: Take action if one point lays outside the $\pm 3 \sigma$ lines.

In general, run rules take advantage of the previously mentioned lines of the Shewhart chart and the number of points that have passed them. The run rules would signal whenever they are satisfied and would not signal otherwise. 


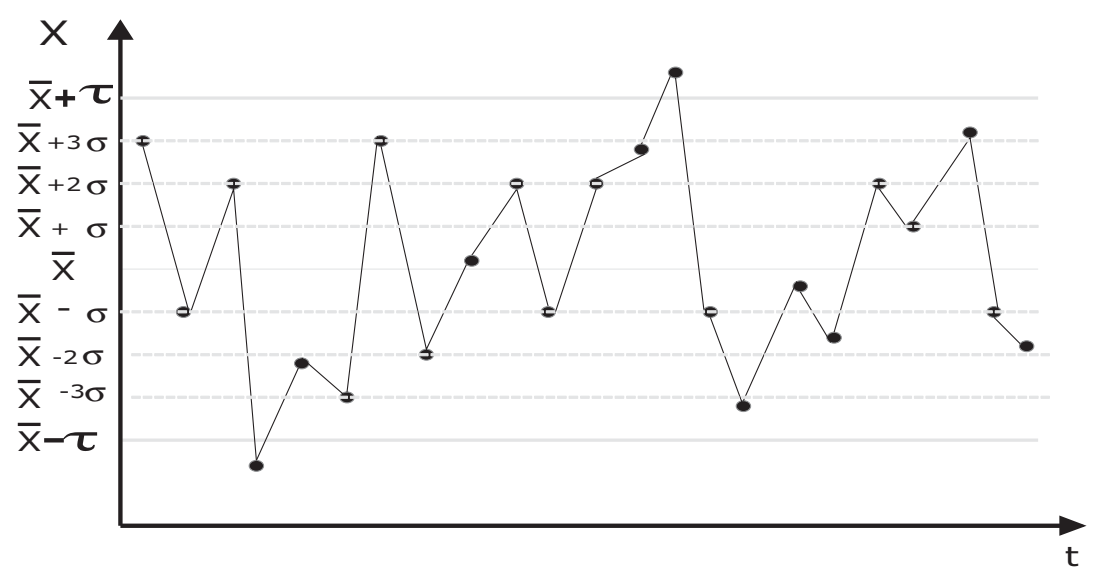

Figure 2: The Shewhart chart.

\section{RESEARCH METHOD}

A flow meter discrepancy (having higher or lower readings than expected) can happen as a result of several factors including:

1. Flow meter drift,

2. A substantial increase or decrease in consumption, and

3. Pipe leakage.

Flow meter drift is often the main reason for flow meter discrepancy in an industrial environment. As for the second reason, a substantial increase in consumption can only be, usually, caused by an addition of a new unit while a substantial decrease in consumption can only be, usually, caused by a removal of a working unit. Each unit would cost tens or hundreds of millions of dollars to buy and install. Therefore, it can be understood that the addition of a new unit or the removal of a working one is a rare event. Consequently, this would make increased (or decreased) flow meter readings due to installing new (or removing old) units a rarity. Pipe leakage is an issue in municipal water systems and not, usually, an issue in industrial water systems because of the high reliability of its pipes. It is safe, therefore, to assume that there are only two factors contributing to the uncertainty of measured flow of a plant (the flow meter reading). These two factors are: (a) the true consumption of the plant which varies with time depending on the production level, the weather, the reliability of plant equipment, etc. and (b) the reliability and accuracy of the flow meter itself.

A dataset of monthly water consumption for an industrial consumer was simulated for this research. The main purpose of the research was to develop rational investigations of drifts so as to have optimal billing with minimal cost and efforts. Also, the modeling frameworks from this research would provide systematic 'alarm' mechanisms to the operation and maintenance staff; especially whenever a flow meter drift might occur in future. Earlier, Ben Salamah et al. [1] developed a seasonal time series consumption mapping (i.e. for a consumption typical of an industrial consumer) with linear patterns and virtual means. Subsequently, the virtual mean mapping was augmented through systematic SPC with CUSUM to rationally detect the drift errors in industrial water metering.

When the input data for the pattern of consumption was simulated, noise (representing the natural randomness within the consuming systems) was added to it. These input data were labeled as normal 
(0) when it was processed by the ANN. Part of these input data had something else besides the normal randomness added to them. In this part of the data, a function causing a drifting process for the data was deliberately inserted. This part of the data was labeled as either drifting upward $(+1)$ or drifting downward $(-1)$ depending on the case. The data labeled drifting upward $(+1)$ represented cases of a drift that produced flow meter readings that are higher than the actual consumption. The data labeled drifting downward (-1) represented cases of a drift that produced flow meter readings that are lower than the actual consumption. Part of the data was saved for testing. This part contained both normal and drifting data of both kinds.

\subsection{The inputs for the artificial neural network}

The ANN used in this research is the three-layer back propagation network. It has an input layer, a hidden layer, and an output layer. The input layer is made up of two parts: a numerical part and a symbolic part. This is similar to what Cheng [2] has done. The purpose of including a numerical part is to study the behavior of the process quantitatively while the purpose of including a symbolic part is to study the behavior of the process qualitatively.

4.1.1 The inputs for the artificial neural network - the numerical inputs

When a process is drifting, the process or quality of interest might take a shape similar to what is shown in Fig. 3. The figure shows a process or quality with an upward drift. Similarly, a downward drift may exist.

In Fig. 3, after the upward drift has started, each point or sample $X_{t}$ is going to be greater than the previous point(s). The opposite would exist if there was a downward drift. Consequently, examining the behavior of $X_{t}$ in relation to its past values might give a clue about the existence of a drift. Hence, an index $b_{i}$ was thought of where

$$
b_{i}=X_{t}-X_{t-i}
$$

This index would numerically compare the current value of the process $X_{t}$ to one of its past values $X_{t-i}$. Comparing the current value of the process with one past value would not be enough. To have a good understanding of the behavior of the process, the current value must be compared with many,

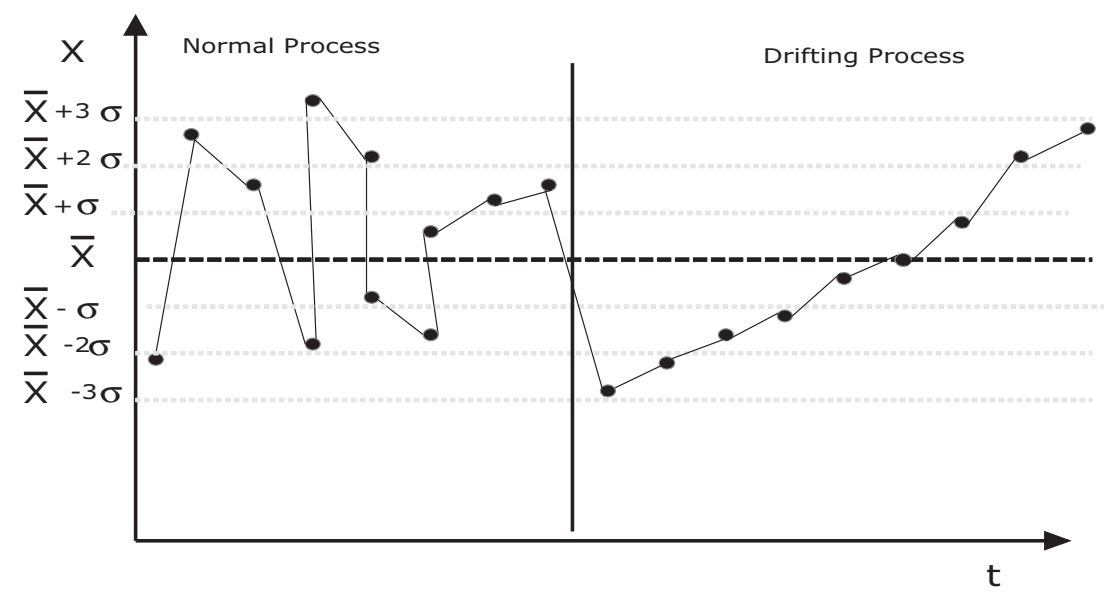

Figure 3: A process with an upward drift. 
$n$, past values. Therefore, for every value of $X_{t}, n$ values of $b_{i}$ are going to be produced. To further explain, for every $X_{t}$ we would have $b_{1}, b_{2}, b_{3}, \ldots, b_{i}, \ldots, b_{n}$.

The $n$ values of the index $b_{i}$ would examine the behavior of the current value of a process in relation to its past values. Nevertheless, the values of $b_{i}$ obtained would be unique to its particular process. The aim of this study is to detect flow meter drift. The pumping station under study has many flow meters. The flow that is measured by one flow meter differs greatly from that measured by other flow meters; sometimes, by several magnitudes. This would mean that each flow meter would require its own simulation which would be exhaustive and computationally expensive. To only make one simulation that is capable of being generalized to many flows (processes), the authors used normalization. The normalized value is computed as follows:

$$
Z_{t}=\frac{X_{t}-\bar{X}}{\sigma}
$$

Where $\bar{X}$ is the mean and $\sigma$ is the standard deviation of the quality or process.

To study the behavior of $Z_{t}$ in relation to its past values an index ${ }^{z} b_{i}$ is defined as:

$$
\begin{aligned}
& { }^{z} b_{i}=Z_{t}-Z_{t-i} \\
& { }^{z} b_{i}=\frac{X_{t}-\bar{X}}{\sigma}-\frac{X_{t-i}-\bar{X}}{\sigma} \quad{ }^{z} b_{i}=\frac{X_{t}-X_{t-i}}{\sigma}
\end{aligned}
$$

Then, for every value of $X_{t}, n$ values of ${ }^{z} b_{i}$ are going to be produced, i.e. for every $X_{t}$ we would have ${ }^{z} b_{1},{ }^{z} b_{2},{ }^{z} b_{3}, \ldots,{ }^{z} b_{i}, \ldots,{ }^{z} b_{n}$. The values of ${ }^{z} b_{i}$ are made for the numerical inputs to the ANN. A question that would rise here is how many values of ${ }^{z} b_{i}$ should be processed by the ANN?

The best number of past values, $n$, to consider as an input to the ANN was an issue addressed by Cheng [2]. He wrote that

'The proposed neural network is based on the assumption that there are a number of observations ready for analysis. The number of data in a sequence provided to the neural network is referred to here as the window size. Since rapid computation is of paramount importance for process control, it is desirable to minimize input layer size to permit efficient computation. The determination of the window size is based on the consideration of Type I error. A small window size might result in a very high Type I error while a large window size may commit a long computation time. In the current research, a window size of 16 was selected through experimentation. The results indicated that further increasing the window size does not provide any significant improvement in performance'.

It is the experience of the authors of this paper that a drift can exist and be unnoticed for a long time. Also, the control in the process under study is done offline and is done in more capable computers than the ones that were available when Cheng first published his paper. The authors of this paper, therefore, decided to make the 'window size' or the number of past values to consider, $n, 17$.

\subsubsection{The inputs for the artificial neural network - the symbolic inputs}

To study the behavior of any process, the numerical characteristics of this behavior would certainly be helpful. The previous sub-section dealt with that; it dealt with the quantitative aspect of the process. A process, nevertheless, can be examined by using a different point of view: The qualitative point of view.

The qualitative point of view would see if the behavior of the process is having certain qualities or not. Because these qualities either exist or not exist or exist in a certain condition, numerical values cannot be assigned to them. Rather, logical or symbolic values would be used for representing them. 
One good method for examining a process qualitatively is to use the previously mentioned run rules. Cheng [2] used one run rule as his symbolic input, namely, the rule when the process exceeded $\bar{X} \pm 3 \sigma$. In the current paper, seven run rules were used as the symbolic inputs to the neural network. The first five run rules were from Koutras et al. [9]. The run rules demand that the process be investigated if

1. One point is outside $\pm 3 \sigma$ lines.

2. Two out of three consecutive points are beyond $\pm 2 \sigma$ lines.

3. Four out of five consecutive points are $\pm 1 \sigma$ or beyond from the mean.

4. Eight consecutive points are on one side of the mean.

5. Six points in a raw steadily increasing or decreasing. The remaining two rules are from Yasui et al. [10].

6. Two of three successive observations exceed $\bar{X} \pm 2.0698 \sigma$.

7. Two successive observations exceed $\bar{X} \pm 1.9322 \sigma$.

Each rule, $R$, would give one of three responses:

1. $R_{1,2, \ldots, 7}=+1$ if the rule is satisfied and the points are greater than the mean.

2. -1 if the rule is satisfied and the points are less than the mean.

3. 0 if the rule is not satisfied.

4.2 The hidden and output layers.

As in any ANN, there are hidden and output layers. This ANN has only one hidden layer. The output layer gives one of three states for the flow meter: normal or upward drifting or downward drifting. These outputs are qualitative or symbolic. Hence, they were represented by three symbols in the simulation. The normal case had the symbol ' 0 ', the upward drifting case had the symbol ' 1 ', and the downward drifting case had the symbol ' -1 '. The structure of the ANN is seen in Fig. 4.

\section{RESULTS OF THE SIMULATION}

One thousand training epochs were performed. The mean squared error (MSE) per epoch for both the training and cross-validation phases are shown in Fig. 5 and Table 1.

A number of data, 1870, was saved for testing. The confusion matrix for the test is shown in Table 2. For the data spared for testing, the confusion matrix shows the performance of the ANN. The confusion matrix compares the actual output with the desired output. It shows how many times the neural network made the right decision and how many times it was 'confused'. For example, there have been a total of 393 cases with an actual upward drift (State (1)). The ANN rightly identified 296 of them as they were, having an upward drift. The network confused 9 cases as having downward drift and 88 cases as being normal while actually they were cases of upward drift.

It can be seen from Table 2 that the ANN developed was correct $89.52 \%$ of the time in determining the status of the flow recorded by a flow meter.

\section{CONCLUSION}

Failing flow meters produce erroneous measurements that disturb the control, organization, regulation, and planning processes for a water system. Previously, work was presented that detected industrial water flow meter drift by the CUSUM method. In this paper, an alternative method called artificial 


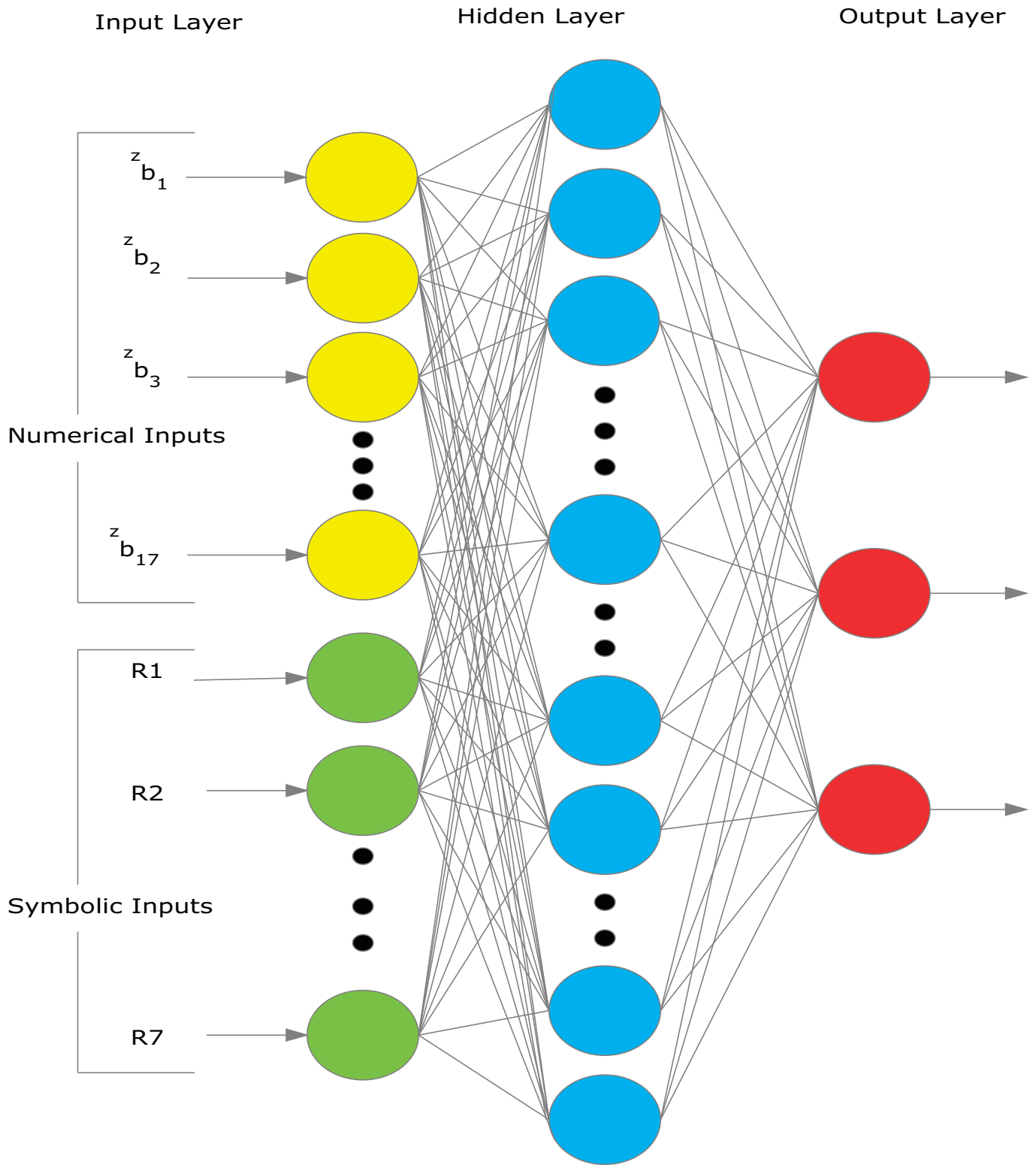

Figure 4: The structure of the artificial neural network.

neural network was introduced. The ANN presented in this paper was the typical three-layer neural network. The input layer was made of 24 inputs. Seven of them were symbolic inputs while the remaining 17 were numerical inputs. The network was trained and tested and the results were satisfactory.

\section{ACKNOWLEDGEMENTS}

The authors would like to thank Prof. Chuen-Sheng Cheng from the Yuan Ze University for his help with this paper. They would also like to thank the two anonymous reviewers for their suggestions. 


\section{MSE versus Epoch}

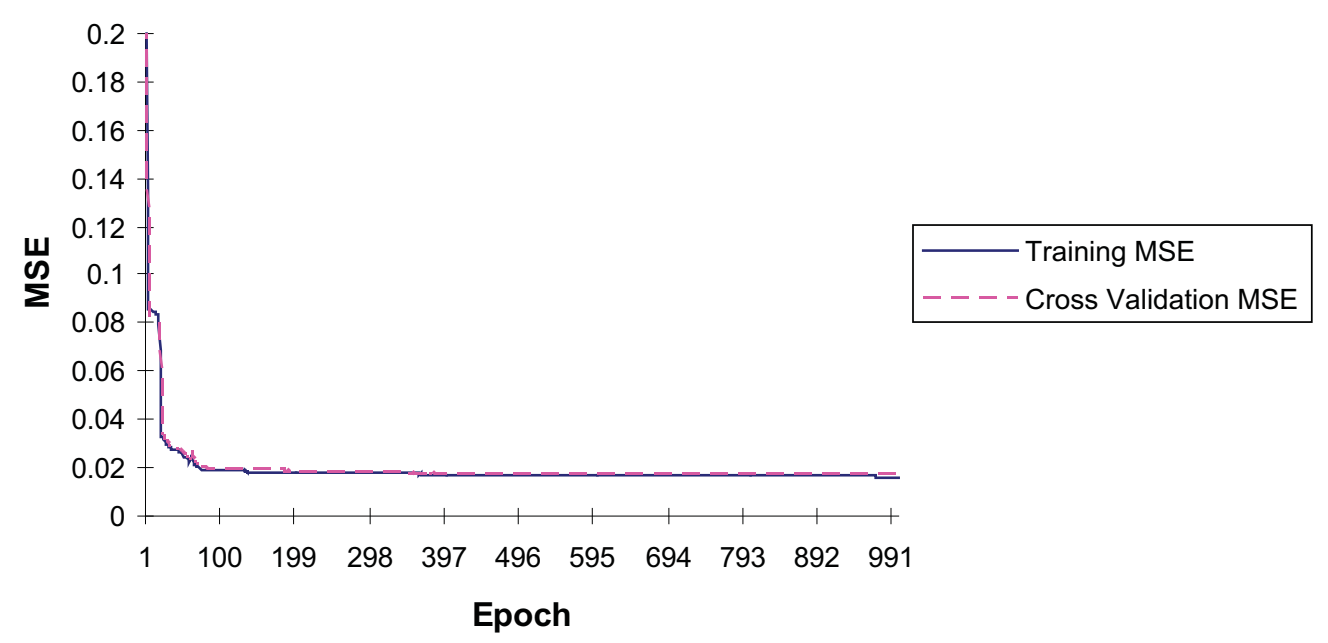

Figure 5: Mean squared error for training and cross-validation.

Table 1: Training results for 1000 epochs.

\begin{tabular}{lcc}
\hline Best networks & Training & Cross-validation \\
\hline Epoch \# & 997 & 968 \\
Minimum MSE & 0.01574738 & 0.016462574 \\
Final MSE & 0.01576385 & 0.017043336 \\
\hline
\end{tabular}

MSE $=$ mean squared error.

Table 2: The confusion matrix.

\begin{tabular}{lcrr}
\hline \multicolumn{4}{c}{ Desired } \\
\hline Output & State $(-1)$ & State $(1)$ & State $(0)$ \\
State $(-1)$ & 266 & 9 & 6 \\
State $(1)$ & 21 & 296 & 13 \\
State $(0)$ & 59 & 88 & 1112 \\
\hline
\end{tabular}

\section{REFERENCES}

[1] Ben Salamah, M., et al., The detection of flow meter drift by using statistical process control. International Journal of Sustainable Development and Planning, To be published.

[2] Cheng, C.S., A multi-layer neural network model for detecting changes in the process mean. Computers \& Industrial Engineering, 28(1), pp. 51-61, 1995. doi:http://dx.doi. org/10.1016/0360-8352(94)00024-H 
[3] Cheng, C.S., A neural network approach for the analysis of control chart patterns. International Journal of Production Research, 35(3), pp. 667-697, 1997. doi:http://dx.doi. org/10.1080/002075497195650

[4] Friedman, M. \& Kandel A., Introduction to pattern recognition: statistical, structural, neural and fuzzy logic approaches. 1 edn. Series in Machine Perception and Artificial Intelligence, eds H. Bunke \& P.S.P. Wang., World Scientific: London, 32, p. 329, 1999.

[5] Bishop, C.M., Neural Networks for Pattern Recognition, Oxford: Oxford University Press, 482,2005

[6] Anagun, A.S., A neural network applied to pattern recognition in statistical process control. Computers \& Industrial Engineering, 35(1-2), pp. 185-188, 1998. doi:http://dx.doi. org/10.1016/S0360-8352(98)00057-6

[7] Hassan, A., et al., Improved SPC chart pattern recognition using statistical features. International Journal of Production Research, 41(7), pp. 1587-1603, 2003.

[8] Pacella, M., Semeraro, Q. \& Anglani, A., Manufacturing quality control by means of a Fuzzy ART network trained on natural process data. Engineering Applications of Artificial Intelligence, 17(1), pp. 83-96, 2004. doi:http://dx.doi.org/10.1016/j.engappai.2003.11.005

[9] Koutras, M.V., Bersimis, S. \& Maravelakis, P.E., Statistical process control using Shewhart control charts with supplementary runs rule. Methodology and Computing in Applied Probability, 9(2), pp. 207-224, 2007. doi:http://dx.doi.org/10.1007/s11009-007-9016-8

[10] Yasui, S., Ojima, Y. \& Suzuki, T., Generalization of the Run Rules for the Shewhart Control Charts in Frontiers in Statistical Quality Control 8, eds H.J. Lenz \& P.T. Wilrich, PhysicaVerlag: New York. pp. 207-219, 2006.

[11] Bowerman, B.L., O'Connell, R.T. \& Koehler, A.B., Forecasting, Time Series and Regression An Applied Approach. Thomson Broks/Cole. p. 686, 2005.

[12] Norton, M., A Quick Course In Statistical Process Control. Technology Skills., Upper Saddle River, New Jersey: Pearson Prentice Hall. p. 56, 2005. 